\title{
Imaging Acquisition Technique Influences Interpretation of Positron Emission Tomography Vascular Activity in Large-Vessel Vasculitis
}

\author{
Kaitlin A. Quinn, MD ${ }^{1,2}$, Joel S. Rosenblum, BS ${ }^{2}$, Casey A. Rimland, PhD $^{2,3}$, K. Bates \\ Gribbons, BS ${ }^{2}$, Mark A. AhIman, MD $^{4}$, Peter C. Grayson, MSc, MD ${ }^{2}$ \\ ${ }^{1}$ Division of Rheumatology, MedStar Georgetown University Hospital, Washington DC, USA \\ ${ }^{2}$ Systemic Autoimmunity Branch, National Institutes of Health, NIAMS, Bethesda, MD, USA \\ ${ }^{3}$ University of North Carolina at Chapel Hill School of Medicine, Chapel Hill, NC, USA \\ ${ }^{4}$ National Institutes of Health, Clinical Center, Radiology and Imaging Sciences, Bethesda, MD, \\ USA
}

\section{Abstract}

Objectives: To determine the impact of imaging acquisition time on interpretation of disease activity on ${ }^{18}$ F-fluorodeoxyglucose positron emission tomography (PET) in large-vessel vasculitis (LVV) and assess the relationship between clinical features and image acquisition time.

Methods: Patients with giant cell arteritis (GCA) and Takayasu's arteritis (TAK) were recruited into a prospective, observational cohort. After a single injection of FDG, all patients underwent two sequential PET scans at one and two-hour time points. Images were interpreted for active vasculitis by subjective assessment, qualitative assessment, and semi-quantitative assessment. Agreement was assessed by percent agreement, Cohen's kappa, and McNemar's test. Multivariable logistic regression identified associations between PET activity and clinical variables.

Results: 79 patients $(\mathrm{GCA}=44, \mathrm{TAK}=35)$ contributed 168 paired one and two-hour PET studies. A total of 94 out of 168 scans (56\%) were interpreted as active at the one-hour time point, and 129 scans $(77 \%)$ were interpreted as active at the two-hour time point $(\mathrm{p}<0.01)$. Associations between clinical variables and PET activity categories (dual inactive, delayed active, dual active) were evaluated. Using multivariable nominal regression, clinically active disease was significantly more common in patients in the delayed active group (Odds Ratio 1.94, 95\% CI $1.13-3.53$; $\mathrm{p}=0.02$ ) and the dual active group (Odds Ratio 1.71, 95\%CI $1.06-2.93 ; \mathrm{p}=0.04$ ) compared to the dual inactive group.

Corresponding Author and Reprint Requests: Kaitlin A. Quinn, MD, National Institutes of Health / NIAMS, 10 Center Drive, Building 10, 10N Rm 311D Bethesda, MD 20892, kaitlin.quinn@nih.gov; Phone: 301-827-9187; Fax: 301-480-3978.

Publisher's Disclaimer: This is a PDF file of an unedited manuscript that has been accepted for publication. As a service to our customers we are providing this early version of the manuscript. The manuscript will undergo copyediting, typesetting, and review of the resulting proof before it is published in its final citable form. Please note that during the production process errors may be discovered which could affect the content, and all legal disclaimers that apply to the journal pertain.

Statements: The authors declare no conflicts of interest. 
Conclusions: Imaging protocol significantly influences interpretation of PET activity in LVV. A substantial proportion of patients with LVV have PET activity only detected by delayed imaging. These patients were significantly more likely to have concomitant clinically-determined active disease.

\section{Keywords}

vasculitis; giant cell arteritis; Takayasu's arteritis; positron emission tomography; imaging

\section{INTRODUCTION}

Giant cell arteritis (GCA) and Takayasu's arteritis (TAK) are the two major forms of largevessel vasculitis (LVV), defined by inflammation involving the aorta and its primary branches ${ }^{1}$. Vascular imaging has become an essential component to complement clinical assessment in patients with LVV. 18F-flurodeoxyglucose (FDG) positron emission tomography (PET) is a type of imaging currently established for use in the diagnosis and monitoring of cancer, but has also been one of several imaging modalities used in the assessment of patients with $\mathrm{LVV}^{2-5}$. Malignant cells are metabolically active and utilize more glucose than other tissues resulting in greater FDG uptake on PET scans. Similarly, abnormal metabolic activity within the walls of large arteries due to activated immune cells can be used as a surrogate for vascular inflammation in $\mathrm{LVV}^{6}$.

The amount of FDG distributed into the arterial wall may be influenced by the timing of image acquisition. In recent guidelines, a minimum of 60 minutes between intravenous FDG administration and image acquisition has been recommended for adequate tracer biodistribution in large-vessel vasculitis ${ }^{67}$. However, in atherosclerosis studies, a longer interval of two hours between FDG administration and image acquisition time has been recommended, to increase time for FDG to distribute into the arterial wall and simultaneously be cleared from the blood pool ${ }^{8}$.

To date, there have been few studies evaluating delayed imaging in $\mathrm{LVV}^{910}$, and it remains uncertain whether the effect of delayed imaging is significant enough to impact the reader's interpretation of disease activity on FDG-PET scans. While delayed imaging may be more sensitive to detect arterial FDG uptake, vascular PET findings at later imaging acquisition times could be increasingly confounded by factors not directly related to active vasculitis, such as atherosclerosis.

The objectives of this study were to: 1) determine the impact of imaging acquisition time on interpretation of disease activity on FDG-PET in large-vessel vasculitis and 2) assess the relationship between clinical features and image acquisition time.

\section{METHODS}

\section{Study population:}

Patients $\geq 18$ years with LVV were recruited into a prospective, observational cohort at the National Institutes of Health (NIH) in Bethesda, MD, USA. Patients fulfilled the 1990 American College of Rheumatology (ACR) Classification Criteria for TAK ${ }^{11}$ or modified 
1990 ACR Criteria for GCA ${ }^{1213}$. Patients could be enrolled at various stages during their disease course.

\section{Clinical Assessment:}

All patients underwent clinical evaluation and imaging at the NIH Clinical Center, with repeat assessments performed at 6-month intervals. The investigative study team performed all clinical assessments 24 hours prior to imaging assessment. Active disease was defined as the presence of any clinical disease features directly attributed to vasculitis at the time of assessment (e.g. headache, carotidynia). Remission was defined as the absence of any clinical symptoms directly attributable to vasculitis. Clinical disease assessment was recorded prior to conducting imaging studies. Additional clinical variables collected included: age, body mass index (BMI), LVV type (TAK vs GCA), glomerular filtration rate (GFR), c-reactive protein (CRP), erythrocyte sedimentation rate (ESR), and treatment status (currently on any treatment vs not on treatment). Treatment could include glucocorticoids, disease-modifying antirheumatic drugs (DMARDs), or biologic therapies.

\section{FDG-PET Imaging Protocol:}

All patients underwent two sequential PET scans on the same day. Following injection, whole-body FDG-PET magnetic resonance imaging (MRI) was performed at one-hour uptake time followed by FDG-PET computed tomography (CT) at two-hours uptake time. Patients were instructed to consume a carbohydrate-sparse meal the day prior to imaging, and fast on the day of imaging. All patients received a single, fixed dose of 10mCi FDG. PET-MRI was performed with a Siemens Biograph mMR (Siemens Medical Solutions, Erlangen, Germany). PET-MRI reconstruction employed MRI-based attenuation correction and iterative reconstruction (172 matrix, $1.2 \mathrm{x}$ zoom, $2.03 \mathrm{~mm}$ slice thickness, point spread function correction, 4.0 Gaussian post-reconstruction filtering). PET-CT was performed with a 128 detector row Siemens Biograph mCT (Siemens Medical Solutions, Erlangen, Germany). Image reconstruction employed $\mathrm{CT}$ attenuation correction and iterative reconstruction (24 iterations, 3 subsets, 256 matrix, $1.2 \mathrm{x}$ zoom, $1.5 \mathrm{~mm}$ slice thickness, timeof-flight, point spread function correction, and no post reconstruction filtering).

\section{FDG-PET Imaging Assessment:}

A single expert with extensive experience in clinical vascular imaging interpretation (MA) reviewed all PET scans included in this study, blinded to clinical data. One-hour and twohour paired studies were read blinded to each other.

Subjective Assessment: Each scan was interpreted as active or inactive vasculitis based on subjective assessment of the reader. Subsequent PET categories were created based upon subjective interpretation of active vasculitis in the paired scans as follows: 1) both time points active (dual active), 2) both time points inactive (dual inactive), 3) only the one-hour time point active (early time point active), and 4) only the two-hour time point active (delayed active).

Qualitative Assessment: The same reader (MA) performed qualitative assessment of FDG uptake in 4 segments of the aorta (ascending, arch, descending thoracic, and 
abdominal) and in 5 branch arteries (innominate, right and left carotids, right and left subclavians). The degree of arterial uptake was visually assessed relative to liver uptake as $0=$ no uptake; $1=$ less than liver uptake; $2=$ same as liver uptake; $3=$ greater than liver uptake. A summary score, PET Vascular Activity Score (PETVAS), was calculated by adding the qualitative scores in the above specified 9 arterial territories, with scores ranging from 0-27 as previously described ${ }^{14}$.

Semi-quantitative Assessment: Arterial FDG uptake was quantified by manually drawing regions of interest (ROI) in OsiriX DICOM Viewer (Version v.9.5.2, Bernex, Switzerland), with attention to both anatomic location on CT or MRI, as appropriate, and coregistered PET activity. In an axial view, ROIs that encompassed both the arterial wall and lumen were drawn around each arterial territory of interest, including five segments of the aorta (ascending, aortic arch, descending, suprarenal abdominal, infrarenal abdominal) and four branch arteries (bilateral carotid and subclavian arteries). This process was repeated for all available image slices to obtain corresponding maximum standardized uptake values $\left(\mathrm{SUV}_{\max }\right)$. The mean $\mathrm{SUV}_{\max }$ for each arterial territory was summed across all the arterial territories and averaged to produce a global summary metric of FDG uptake for the entire aorta and branch arteries of interest $\left(\mathrm{SUV}_{\text {sum }}\right)$.

\section{Statistical Analysis:}

A random sampling of 15\% imaging studies at both one and two-hour time points were reassessed by the same reader on different days. Intra-rater agreement for reader interpretation of disease activity was assessed by cohen's kappa. Agreement between one and two-hour PET activity was assessed by percent overall agreement, Cohen's kappa, and McNemar's test. PETVAS was compared across groups of patients categorized by image interpretation using the Kruskal-Wallis test with post-hoc Dunn's test for multiple comparisons. Nominal logistic regression, using - both scans inactivell as the reference group, was used to study the associations between PET activity categories and the following predictor variables: age, BMI, gender, LVV type (TAK vs GCA), clinical status (active vs remission), ESR, CRP, and treatment status (yes vs no). Only variables with $p<0.1$ in univariable models were included in multivariable regression. $\mathrm{SUV}_{\text {sum }}$, derived by semiquantitative data, was compared between the one and two-hour PET scans using MannWhitney test and Spearman correlation. Percent change in SUV $\mathrm{Sum}_{\text {sum }}$ between the one and two-hour PET scans stratified by clinical disease activity was calculated using MannWhitney test.

\section{Ethics and Informed Consent:}

All patients provided written informed consent. An institutional review board and radiation safety committee at the NIH approved the research.

\section{RESULTS}

\section{Study Population:}

A total of 79 patients were recruited into the study, including 44 patients with GCA and 35 patients with TAK. There were 168 paired one and two-hour PET studies performed, as 
some patients underwent multiple paired one and two-hour PET studies at 6-month intervals. Baseline demographics of the study population are shown in Table 1. Patients with TAK were younger than patients with GCA, as implied in the definitions of the diseases, and patients with TAK had longer disease duration compared to patients with GCA.

\section{Subjective Assessment of Disease Activity:}

There was substantial intra-rater agreement (kappa=0.76, CI 0.56-0.96) for the subjective assessment of disease activity. A total of 94 out of 168 scans (56\%) were interpreted as active at the one-hour time point, and 129 scans (77\%) were interpreted as active at the twohour time point $(\mathrm{p}<0.01)$ (Table 2). PET studies were interpreted as active at both the onehour and two-hour imaging time points in 90 cases and inactive at both time points in 35 cases (concordance $=74 \%$, kappa $=0.45$ indicating fair agreement). In cases where the PET was interpreted as active at only one time point, scans were more likely to be interpreted as active at the delayed time point compared to the early time point (39 vs 4 cases, $\mathrm{p}<0.01$ ). Increased activity at delayed imaging was seen in both TAK and GCA (Supplementary Table 1). A representative image from a patient with activity only at the delayed time point is shown in Figure 1.

Patients with scans active only at the one-hour time point had a greater BMI than other patients (median BMI: dual active group=27.6; dual inactive group=27.8; early active group $=35.8$; delayed active group $=25.5 ; \mathrm{p}=0.03$ ). There were no other clinical features that defined the patients with disease activity only at the one-hour time point. Due to small number of scans active only at the early time point, this group was excluded from further analysis.

\section{Qualitative Assessment of Disease Activity:}

PET activity was next assessed at the one and two-hour time points using the PETVAS. The median PETVAS was greater at the two-hour imaging time point than the one-hour time point (19 vs 16, $\mathrm{p}<0.01)$. The greatest change in PETVAS between the one and two-hour imaging time points was seen in the delayed active group, where the median PETVAS increased by 8 points from one to two hours ( $11 \mathrm{vs} 19, \mathrm{p}<0.01)$. In this delayed active group, the median PETVAS at one hour was similar to the PETVAS at one hour in the dual inactive group (11 vs 11.5, p>0.99), and the median PETVAS at two hours was similar to the PETVAS at two hours in the dual active group (19 vs 22, $\mathrm{p}=0.07$ ) as shown in Figure 2.

\section{Association of PET and Clinical Features:}

The association of clinical interpretation and PET interpretation of disease activity was studied at each time point. Clinical assessment was significantly associated with interpretation of PET scan activity at the 2-hour time point $(\mathrm{p}=0.01)$ but not the 1-hour time point ( $\mathrm{p}=0.06)$ (Supplementary Table 2).

Associations between clinical variables and PET activity categories (dual inactive, delayed active, dual active) were evaluated. In univariable analyses, CRP, gender, LVV type, and clinically active disease were significantly associated with PET activity category, whereas age, BMI, ESR, and treatment status (yes vs. no) were not. 
Using multivariable nominal regression, clinically active disease was significantly more common in patients in the delayed active group (Odds Ratio 1.94, 95\% CI 1.13 - 3.53; $\mathrm{p}=0.02$ ) and the dual active group (Odds Ratio 1.71, 95\%CI 1.06 - 2.93; $\mathrm{p}=0.04$ ) compared to the dual inactive group (Table 3 ). CRP, gender, and LVV type were no longer significantly associated with PET category in multivariable analysis.

\section{Semi-Quantitative Assessment of Disease Activity}

Disease activity was assessed semi-quantitatively by calculating the average FDG uptake in the arteries $\left(\mathrm{SUV}_{\text {sum }}\right)$ at the one and two-hour imaging time points. Across all studies, SUV $_{\text {sum }}$ was significantly greater at two hours compared to one hour ( $3.1 \pm 1.0$ vs $2.3 \pm 0.4$; $\mathrm{p}<0.01$ ) (Figure $3 \mathrm{~A}$ ). However, there was strong correlation between $\mathrm{SUV}_{\text {sum }}$ at both time points $\left(r=0.83, p<0.01\right.$ ), indicating that although $\mathrm{SUV}_{\text {sum }}$ was greater at the delayed time point, FDG arterial uptake was detected in the same arterial territories at both the one and two-hour time points (Figure 3B). When patients were stratified by clinical disease status (i.e. clinically active versus remission), patients with clinically active disease had a greater percent change in $\mathrm{SUV}_{\text {sum }}$ from the one to two-hour time points than patients in clinical remission ( $40 \%$ vs $32 \%, \mathrm{p}<0.01$ ) (Figure 3C).

\section{DISCUSSION}

This study demonstrates that imaging acquisition time significantly influences reader interpretation of disease activity in PET scans performed in patients with LVV. Delayed imaging results in increased FDG uptake into the arterial wall and increases the likelihood that a PET scan will be interpreted as active vasculitis. When images were obtained one hour after FDG injection, 56\% of the scans (94/168 scans) were interpreted as active vasculitis, and this percentage increased to $77 \%$ (129/168 scans) when the same patients underwent delayed imaging two hours after injection of FDG. Importantly, delayed imaging resulted in the identification of a subset of patients (23\%) who had evidence of active vasculitis visualized only at the two-hour time point. Similar to patients with active vasculitis at both the one and two-hour time points, the subset of patients with active disease only on delayed imaging was more likely to have associated clinically active disease compared to patients without evidence of vasculitis at either time point.

Consistent with prior reports and recommendations in atherosclerosis, findings from this study support delayed FDG-PET image acquisition in LVV. Compared to PET imaging in oncology where a one-hour time point is sufficient, delayed imaging allows time for FDG distribution into the arterial wall with simultaneous elimination from blood pool ${ }^{815-18}$. Recent guidelines for FDG-PET assessment in LVV recommend at least 60 minutes, and preferably 90 minutes, image acquisition time but acknowledge limited evidence to support these recommendations ${ }^{619}$. A previous study prospectively evaluated early acquisition (60 minutes) versus delayed imaging (180 minutes) in the evaluation of FDG-PET in twentythree patients with LVV and concluded that delayed imaging led to improved visualization of the arterial wall ${ }^{10}$. However, this study did not assess how delayed imaging affected reader interpretation of vascular PET activity, nor did it investigate the clinical features of patients who had increased PET activity at later imaging time points. 
This study underscores a need for the standardization of imaging acquisition time in LVV, as FDG-PET plays an increased role in the diagnosis and monitoring of disease activity in LVV. Because imaging acquisition time influences interpretation of vascular activity on FDG-PET, standardization of acquisition time will be necessary for comparison of studies over time and across different cohorts. Standardization of imaging acquisition time will be particularly important if FDG-PET is incorporated as an outcome measure in future clinical trials in LVV.

Qualitative and semi-quantitative assessment strategies employed in this study corroborate that delayed imaging results in increased arterial FDG uptake, further explaining why scans at delayed time points are more likely to be interpreted as active vasculitis. Analyses that used PETVAS, a qualitative summary score of arterial FDG uptake, highlighted a subset of patients who had an increased burden of vascular activity only at later imaging time points. Disease activity in these patients would have been missed without delayed imaging, as PETVAS at one hour in this subset of patients did not differ from PETVAS in patients with no evidence of PET activity at either one or two hours. Beyond qualitative assessment, a significantly greater amount of arterial FDG uptake was measured by semi-quantitative approaches at the two-hour compared to one-hour time point, especially among patients with clinically active disease.

Several limitations of this study are considered. This was a single-center study, necessitating reproducibility of these findings in other cohorts. PET scans were only interpreted by a single reader, but intra-rater agreement was excellent and all studies were read blinded to each other. This study also did not include a control group to compare PET scans at onehour and two-hour time points in patients who did not have LVV. To minimize radiation, patients underwent serial imaging on two different imaging platforms, with FDG-PET MRI at the one-hour imaging acquisition time and FDG-PET CT at the two-hour imaging acquisition time. FDG uptake on PET-CT and PET-MRI are strongly correlated in oncologic populations ${ }^{2021}$, LVV populations ${ }^{22}$, and in this study. Nevertheless, imaging platform may have influenced the subjective interpretation of the studies and the quantification of arterial FDG uptake. Although arterial FDG uptake was consistently greater at the two-hour PET-CT than the one-hour PET-MRI, specific subsets of patients demonstrated a proportionally greater amount of change in arterial FDG uptake over time, highlighting the importance of imaging acquisition time beyond imaging platform.

Additionally, compared to patients in clinical remission, patients with clinically active disease had a proportionally greater increase in arterial FDG uptake from one to two hours, emphasizing the clinical importance of imaging acquisition time on the distribution of FDG into the artery in specific patients with LVV.

In conclusion, findings from this study demonstrate that imaging protocol significantly influences interpretation of FDG-PET activity in LVV. A substantial proportion of patients with LVV have PET activity detected by delayed-imaging that would have been missed on conventional one-hour imaging studies. Importantly, these patients with activity detectable only by delayed-imaging were significantly more likely to have concomitant clinically- 
determined active disease. Future studies of the role of FDG-PET in LVV should employ delayed imaging acquisition protocols.

\section{Supplementary Material}

Refer to Web version on PubMed Central for supplementary material.

\section{Acknowledgments and affiliations:}

This work was supported by the Intramural Research Program at the National Institute of Arthritis and Musculoskeletal and Skin Diseases. KQ received funding from a Vasculitis Clinical Research Consortium (VCRC) / Vasculitis Foundation (VF) Fellowship. The Vasculitis Clinical Research Consortium (VCRC) is part of the Rare Diseases Clinical Research Network (RDCRN), an initiative of the Office of Rare Diseases Research (ORDR), National Center for Advancing Translational Science (NCATS). The VCRC is funded through collaboration between NCATS, and the National Institute of Arthritis and Musculoskeletal and Skin Diseases (U54 AR057319).

\section{References}

1. Jennette JC, Falk RJ, Bacon PA, et al. 2012 revised International Chapel Hill Consensus Conference Nomenclature of Vasculitides. Arthritis Rheum 2013;65:1-11. [PubMed: 23045170]

2. Blockmans D, Bley T, Schmidt W. Imaging for large-vessel vasculitis. Curr Opin Rheumatol 2009;21:19-28. [PubMed: 19077714]

3. Cimmino MA, Camellino D. Large vessel vasculitis: which imaging method? Swiss Med Wkly 2017;147:w14405. [PubMed: 28322419]

4. Prieto-Gonzalez S, Depetris M, Garcia-Martinez A, et al. Positron emission tomography assessment of large vessel inflammation in patients with newly diagnosed, biopsy-proven giant cell arteritis: a prospective, case-control study. Ann Rheum Dis 2014;73:1388-92. [PubMed: 24665112]

5. Lee YH, Choi SJ, Ji JD, et al. Diagnostic accuracy of 18F-FDG PET or PET/CT for large vessel vasculitis : A meta-analysis. Z Rheumatol 2016;75:924-31. [PubMed: 26704559]

6. Slart R, Writing g, Reviewer g, et al. FDG-PET/CT(A) imaging in large vessel vasculitis and polymyalgia rheumatica: joint procedural recommendation of the EANM, SNMMI, and the PET Interest Group (PIG), and endorsed by the ASNC. Eur J Nucl Med Mol Imaging 2018;45:1250-69. [PubMed: 29637252]

7. Jamar F, Buscombe J, Chiti A, et al. EANM/SNMMI guideline for 18F-FDG use in inflammation and infection. J Nucl Med 2013;54:647-58. [PubMed: 23359660]

8. Bucerius J, Hyafil F, Verberne HJ, et al. Position paper of the Cardiovascular Committee of the European Association of Nuclear Medicine (EANM) on PET imaging of atherosclerosis. Eur J Nucl Med Mol Imaging 2016;43:780-92. [PubMed: 26678270]

9. Martinez-Rodriguez I, Martinez-Amador N, Banzo I, et al. Assessment of aortitis by semiquantitative analysis of 180-min 18F-FDG PET/CT acquisition images. Eur J Nucl Med Mol Imaging 2014;41:2319-24. [PubMed: 25056634]

10. Martinez-Rodriguez I, del Castillo-Matos R, Quirce R, et al. Comparison of early (60 min) and delayed (180 min) acquisition of 18F-FDG PET/CT in large vessel vasculitis. Rev Esp Med Nucl Imagen Mol 2013;32:222-6. [PubMed: 23688731]

11. Arend WP, Michel BA, Bloch DA, et al. The American College of Rheumatology 1990 criteria for the classification of Takayasu arteritis. Arthritis Rheum 1990;33:1129-34. [PubMed: 1975175]

12. Hunder GG, Bloch DA, Michel BA, et al. The American College of Rheumatology 1990 criteria for the classification of giant cell arteritis. Arthritis Rheum 1990;33:1122-8. [PubMed: 2202311]

13. Langford CA, Cuthbertson D, Ytterberg SR, et al. A Randomized, Double-Blind Trial of Abatacept (CTLA-4Ig) for the Treatment of Giant Cell Arteritis. Arthritis Rheumatol 2017;69:837-45. [PubMed: 28133925]

14. Grayson PC, Alehashemi S, Bagheri AA, et al. (18) F-Fluorodeoxyglucose-Positron Emission Tomography As an Imaging Biomarker in a Prospective, Longitudinal Cohort of Patients With Large Vessel Vasculitis. Arthritis Rheumatol 2018;70:439-49. [PubMed: 29145713] 
15. Blomberg BA, Akers SR, Saboury B, et al. Delayed time-point 18F-FDG PET CT imaging enhances assessment of atherosclerotic plaque inflammation. Nucl Med Commun 2013;34:860-7. [PubMed: 23799532]

16. Bucerius J, Mani V, Moncrieff C, et al. Optimizing 18F-FDG PET/CT imaging of vessel wall inflammation: the impact of $18 \mathrm{~F}-\mathrm{FDG}$ circulation time, injected dose, uptake parameters, and fasting blood glucose levels. Eur J Nucl Med Mol Imaging 2014;41:369-83. [PubMed: 24271038]

17. Tawakol A, Migrino RQ, Bashian GG, et al. In vivo 18F-fluorodeoxyglucose positron emission tomography imaging provides a noninvasive measure of carotid plaque inflammation in patients. $\mathbf{J}$ Am Coll Cardiol 2006;48:1818-24. [PubMed: 17084256]

18. Rudd JH, Warburton EA, Fryer TD, et al. Imaging atherosclerotic plaque inflammation with [18F]fluorodeoxyglucose positron emission tomography. Circulation 2002;105:2708-11. [PubMed: 12057982]

19. Dejaco C, Ramiro S, Duftner C, et al. EULAR recommendations for the use of imaging in large vessel vasculitis in clinical practice. Ann Rheum Dis 2018;77:636-43. [PubMed: 29358285]

20. Heusch P, Buchbender C, Beiderwellen K, et al. Standardized uptake values for [(1)(8)F] FDG in normal organ tissues: comparison of whole-body PET/CT and PET/MRI. Eur J Radiol 2013;82:870-6. [PubMed: 23394765]

21. Afaq A, Fraioli F, Sidhu H, et al. Comparison of PET/MRI With PET/CT in the Evaluation of Disease Status in Lymphoma. Clin Nucl Med 2017;42:e1-e7. [PubMed: 27607161]

22. Einspieler I, Thurmel K, Pyka T, et al. Imaging large vessel vasculitis with fully integrated PET/ MRI: a pilot study. Eur J Nucl Med Mol Imaging 2015;42:1012-24. [PubMed: 25876704] 
A.

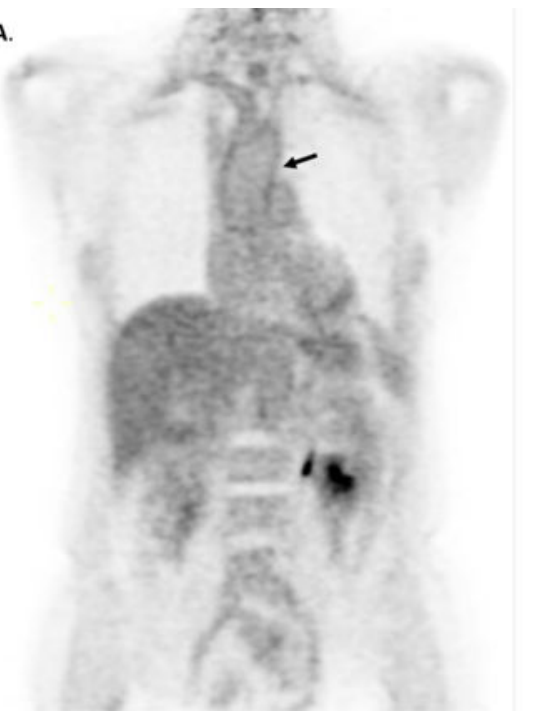

B.

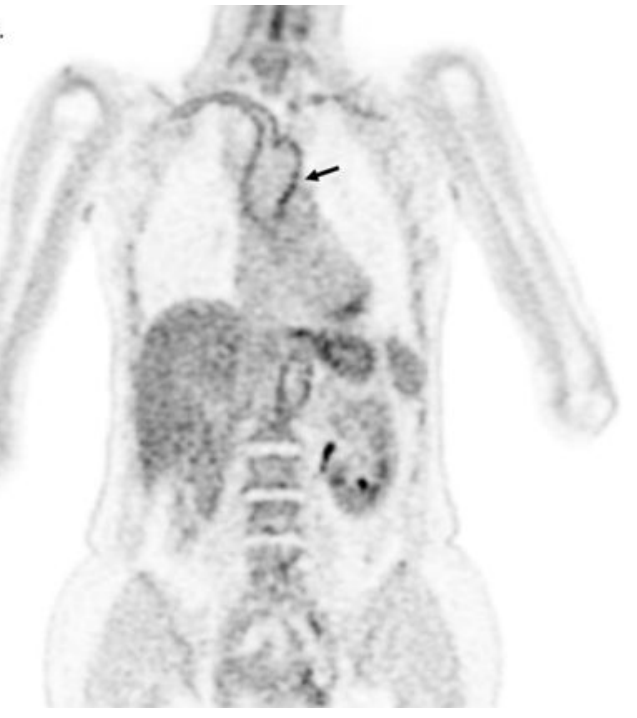

Figure 1: Representative Abnormal ${ }^{18}$ F-fluorodeoxyglucose Positron Emission Tomography (PET) at Delayed Imaging Timepoint.

A 64 year-old Caucasian female with biopsy proven giant cell arteritis underwent a PETMRI at the one-hour time point followed by a PET-CT at the two-hour time point. There is significantly more FDG uptake in the wall of the aorta (arrow) and clearance of FDG from blood pool at the two-hour time point (B) compared to the one-hour time point (A). 


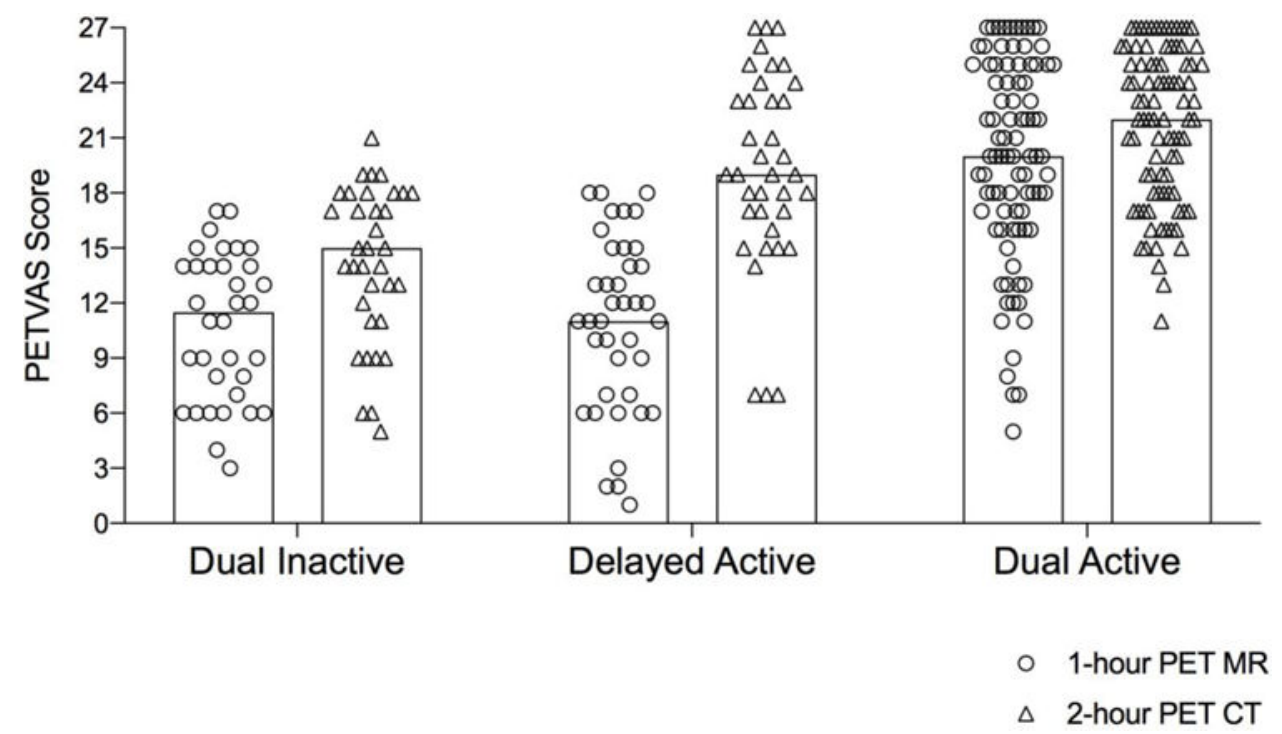

Figure 2: Qualitative Assessment Using PET Vascular Activity Score (PETVAS) at One and TwoHour Imaging Timepoints in Large-Vessel Vasculitis.

Patients were divided into PET activity categories using subjective interpretation of activity of paired imaging studies as 1) dual inactive, 2) delayed active, and 3) dual active. PETVAS was calculated by adding qualitative assessment scores in 9 arterial territories (scale 0-27). PETVAS at both one and two-hour time points in each category is displayed. Median PETVAS was greater at the two-hour imaging time point than the one-hour time point in each category, but the greatest change in PETVAS between the one and two-hour time points is seen in the delayed active group. 
A.

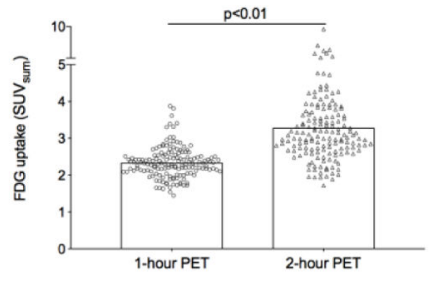

B.

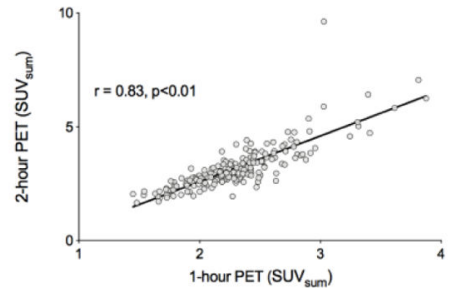

C.

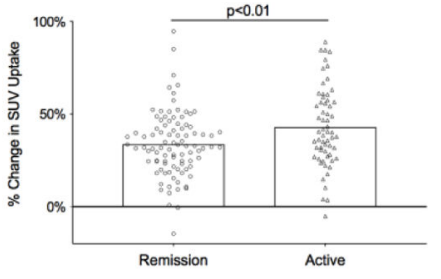

Figure 3: Semi-Quantitative Assessment of Arterial FDG Uptake at One and Two-Hour Imaging Timepoints in Large-Vessel Vasculitis.

Average FDG uptake in the arteries $\left(\mathrm{SUV}_{\text {sum }}\right)$ was semi-quantitatively measured at the one and two-hour imaging time points. $\mathrm{SUV}_{\text {sum }}$ was significantly greater at two hours compared to one hour (A), but there was an overall strong correlation at both time points (B). The change in $\mathrm{SUV}_{\text {sum }}$ between one and two-hour time points was greatest in patients who had clinically active disease compared to patients in remission $(\mathbf{C})$. 
Table 1:

Study Population Baseline Demographics

\begin{tabular}{|c|c|c|c|}
\hline & All Patients $n=79$ & GCA $n=44$ & TAK $\mathbf{n}=35$ \\
\hline \multicolumn{4}{|l|}{ Paired 1 and 2-hour PET studies } \\
\hline Total (n) & 168 & 107 & 61 \\
\hline 1 study visit & 37 & 17 & 20 \\
\hline 2 study visits & 18 & 10 & 8 \\
\hline$\geq 3$ study visits & 24 & 17 & 7 \\
\hline Age (Years $\pm S D)$ & $54.2 \pm 20.3$ & $69.8 \pm 9.0$ & $35.1 \pm 12.2$ \\
\hline Gender (\% female) & $60(76 \%)$ & $34(77 \%)$ & $26(74 \%)$ \\
\hline $\mathrm{BMI}( \pm S D)$ & $26.3 \pm 5.0$ & $26.7 \pm 4.2$ & $25.9 \pm 5.8$ \\
\hline Disease duration (Years $\pm S D$ ) & $6.0 \pm 8.6$ & $2.4 \pm 2.6$ & $10.7 \pm 11.1$ \\
\hline
\end{tabular}

$\mathrm{PET}={ }^{18}$ F-flurodeoxyglucose positron emission tomography, $\mathrm{SD}=$ standard deviation, $\mathrm{GCA}=$ Giant cell arteritis, TAK=Takayasu's arteritis, BMI=body mass index. Patients underwent paired 1 and 2-hour PET studies at each study visit 
Table 2:

Assessment of Disease Activity by Global Impression

\begin{tabular}{lcccc}
\hline & & \multicolumn{2}{c}{ 2-hour PET } & \\
\cline { 3 - 4 } & & Active & Inactive & Total \\
\cline { 2 - 5 } 1-hour PET & Active & 90 & 4 & 94 \\
& Inactive & 39 & 35 & 74 \\
& Total & 129 & 39 & 168 \\
\hline
\end{tabular}

$\mathrm{PET}={ }^{18}$ F-flurodeoxyglucose positron emission tomography 
Table 3:

Clinical Features Associated with PET Activity Categories

\begin{tabular}{llcc}
\hline Predictor Variable & Odds ratio & 95\% Confidence Interval & P-value \\
\hline Dual active & & & \\
\hline Clinically active disease & 1.71 & $1.06-2.93$ & 0.04 \\
CRP (per 1mg/L) & 1.05 & $1.00-1.14$ & 0.12 \\
Female gender & 1.13 & $0.68-1.85$ & 0.62 \\
LVV type (GCA) & 1.33 & $0.86-2.04$ & 0.19 \\
\hline Delayed active only & & & \\
\hline Clinically active disease & 1.94 & $1.13-3.53$ & 0.02 \\
CRP (per 1mg/L) & 1.01 & $0.92-1.08$ & 0.88 \\
Male gender & 1.67 & $1.00-2.86$ & 0.05 \\
LVV type (TAK) & 1.31 & $0.81-2.14$ & 0.28 \\
\hline
\end{tabular}

$\mathrm{CRP}=\mathrm{C}$ reactive protein, $\mathrm{LVV}=$ large-vessel vasculitis, $\mathrm{TAK}=$ Takayasu's arteritis, $\mathrm{GCA}=$ Giant cell arteritis 